\title{
BMJ Open PROphylactic MESH (PROMESH) for stoma closure: does it reduce the incidence of incisional hernia? Protocol for a triple-blinded randomised controlled trial
}

Jeremy Meyer (10 , ${ }^{1,2}$ Vaihere Delaune, ${ }^{1,2}$ Ziad Abbassi, ${ }^{1,2}$ Jonathan Douissard, ${ }^{1,2}$ Christian Toso, ${ }^{1,2}$ Frédéric Ris, ${ }^{1,2}$ Nicolas Buchs ${ }^{1,2}$

To cite: Meyer J, Delaune V, Abbassi Z, et al. PROphylactic MESH (PROMESH) for stoma closure: does it reduce the incidence of incisional hernia? Protocol for a triple-blinded randomised controlled trial. BMJ Open 2021;11:e053751. doi:10.1136/ bmjopen-2021-053751

- Prepublication history for this paper is available online. To view these files, please visit the journal online (http://dx.doi. org/10.1136/bmjopen-2021 053751).

Received 28 June 2021 Accepted 19 November 2021

Check for updates

(C) Author(s) (or their employer(s)) 2021. Re-use permitted under CC BY-NC. No commercial re-use. See rights and permissions. Published by BMJ.

${ }^{1}$ Medical School, University of Geneva, Geneva, Switzerland

${ }^{2}$ Division of Digestive Surgery, University Hospitals of Geneva, Geneva, Switzerland

Correspondence to Dr Jeremy Meyer; jeremy.meyer@hcuge.ch

\section{ABSTRACT}

Introduction Application of a prophylactic mesh during stoma closure was shown to reduce the incidence of incisional hernia at the site of stoma closure. Our objective is to provide high quality evidence to validate this finding.

Methods and analysis The study will be a randomised controlled triple-blinded superiority parallel monocentric trial. Patients undergoing elective ileostomy or colostomy closure after surgery for digestive cancer will be eligible for inclusion. Patients allergic to the mesh, immunosuppressed or refusing to participate will be excluded. Randomisation will be performed based on a 1:1 allocation ratio between stoma closure with application of a non-absorbable mesh in the sublay position (intervention) and stoma closure without a mesh (control). The primary outcome will be the 1-year incidence of incisional hernia at the site of stoma closure, determined clinically and by CT. Secondary outcomes will be the 31-day incidence of surgical site infection and the modified Carolinas Comfort Scale. Patients, radiologists and investigators performing the assessment at 1 year will be blinded for the allocated study group. Analysis will be performed in intention-to-treat. The trial will include 68 patients (34 with mesh, 34 without mesh).

Ethics and dissemination The present randomised controlled trial was registered into clinicaltrials. gov (NCT 04510558) and was accepted by the local ethic committee (Geneva, Switzerland: CCER 2021 00053). The results will be presented at national and international congresses in the fields of colorectal surgery and general surgery, and published in a peerreviewed journal.

\section{BACKGROUND}

Recent evidence reporting on the incidence of incisional hernia after stoma closure is limited by an important heterogeneity. ${ }^{1-4}$ The most frequently reported average incidence is of $30 \%$, and the highest incidence is reported after colostomy closure. ${ }^{1-4}$
Strengths and limitations of this study

Good external validity due to the representation of different types of lower gastrointestinal tract stomas.

- Application of an affordable mesh in extraperitoneal position.

- Assessment of the presence or not of incisional hernia at 1 year by CT.

- Pooling of all types of stomas, which have slightly different incidences of incisional hernia after closure.

- Monocentre design, which may affect recruitment rate and external validity.

Incisional hernia at site of stoma leads to patient's discomfort, quality of life impairment ${ }^{5}$ life-threatening complications such as bowel obstruction, and increased costs for healthcare systems. ${ }^{6-8}$

Several retrospective studies have investigated the impact of the application of a prophylactic mesh during stoma closure on the incidence of incisional hernia. For instance, Liu et al retrospectively compared 47 patients who benefited from ileostomy closure with a prophylactic synthetic non-resorbable mesh to 36 patients who underwent standard closure, and found that incidences of incisional hernia (with a median follow-up of 18 months) were of $6.4 \%$ and $36.1 \%$, respectively. ${ }^{9}$ Similarly, a retrospective casecontrol study compared 30 patients who had ileostomy closure with prophylactic biological mesh with 30 matched patients without mesh. At 1 year, the incidence of incisional hernia diagnosed by CT was of $3.3 \%$ in patients with mesh versus $40 \%$ in patients without mesh. ${ }^{10}$ More recently, the ROCSS (Reinforcement of Closure of Stoma Site) trial showed that applying a 
biological mesh reduced the incidence of clinically detectable incisional hernia from $20 \%$ to $12 \%$ after 2 years of follow-up, and the incidence of radiologicallydetectable incisional hernia from $21 \%$ to $9 \%$ after 1 year of follow-up. ${ }^{11}$ However, the mesh was applied intra-abdominally, which might potentially result in long-term complications due to bowel adhesion to the abdominal wall. Further, biologic meshes are expensive, which might limit the adoption of the technique, and their resorbable nature might limit their efficiency. Better long-term results could potentially be achieved with non-resorbable meshes.

Therefore, we have planned to undertake a randomised controlled trial (RCT) aiming at determining whether application of a prophylactic nonresorbable mesh in the sublay position allows or not reducing the incidence of incisional hernia after ileostomy or colostomy closure.

\section{METHODS/DESIGN}

\section{Reporting of the trial}

The Standard Protocol Items: Recommendations for Interventional Trials statement was followed ${ }^{12}$ for elaboration of the trial protocol. Reporting of the results will be performed according to the Consolidated Standards of Reporting Trials statement. ${ }^{13}$

\section{Study design}

The study will be a randomised controlled superiority triple-blinded monocentric trial with parallel groups determining the incidence of incisional hernia in patients undergoing ileostomy or colostomy closure with or without prophylactic mesh.

\section{Study setting}

The study will take place at the Division of Digestive Surgery, University Hospitals of Geneva, Geneva, Switzerland.

\section{Population}

Adult patients undergoing ileostomy or colostomy closure after surgery for digestive cancer will be considered as eligible for the study.

\section{Inclusion criteria}

- Adult patient.

- Ileostomy or colostomy created during surgery for digestive cancer.

- Planned elective closure of ileostomy or colostomy.

- Informed written consent.

\section{Exclusion criteria}

- Personal history of allergy to one of several components of the mesh.

- Patients under systemic corticosteroids or other systemic immunosuppressive treatment (not stopped at least 1 month before surgery).
- Inability/refusal to follow the procedures of the study.

\section{Drop-outs}

- Stoma closure not performed.

- One-year follow-up not completed.

- Withdrawal of consent.

- Violation of protocol.

Intervention: stoma closure with non-resorbable mesh in the sublay position

- Antibioprophylaxis using cefuroxime and metronidazole.

- Standard disinfection and sterile draping.

- Circular incision around the stoma using the scalpel blade.

- Dissection around the stoma until entering the abdominal cavity.

- Closure of the stoma using a linear stapler.

- A midline laparotomy may be performed or not.

- Extra-corporeal side-to-side handsewn anastomosis or stapled end-to-end anastomosis (for colorectal anastomosis).

- Closure of the posterior rectus sheath using interrupted Maxon 2-0 stitches.

- Retromuscular application of an ULTRAPRO Advanced Mesh, Ethicon, Johnson \& Johnson, with at least $+2 \mathrm{~cm}$ overlap on the edges, secured by 4 stitches of Maxon 2-0.

- Closure of the anterior rectus sheath using interrupted Maxon 2-0 stitches.

- No subcutaneous stitches.

- Partial purse-string skin closure of stoma site using Monocryl 3-0.

- Standard wound dressing (no prophylactic negativepressure wound therapy).

\section{Control: stoma closure without mesh}

- Antibioprophylaxis using cefuroxime and metronidazole.

- Standard disinfection and sterile draping.

- Circular incision around the stoma using the scalpel blade.

- Dissection around the stoma until entering the abdominal cavity.

- Closure of the stoma using a linear stapler.

- A midline laparotomy may be performed or not.

- Extracorporeal side-to-side handsewn anastomosis or stapled end-to-end anastomosis (for colorectal anastomosis).

- Closure of the posterior rectus sheath using interrupted Maxon 2-0 stitches.

- Closure of the anterior rectus sheath using interrupted Maxon 2-0 stitches.

- No subcutaneous stitches.

- Partial purse-string skin closure of stoma site using Monocryl 3-0. 
- Standard wound dressing (no prophylactic negativepressure wound therapy).

Patient and public involvement

No patient was involved in the conception of this study.

\section{OUTCOMES}

\section{Primary outcome}

The primary outcome will be the incidence of incisional hernia at the site of stoma closure at 1 year after closure. The presence or absence of an incisional hernia will be assessed clinically by a consultant general surgeon in the upright and supine positions, at rest and during Valsalva manoeuvre, both visually and by digital palpation. The presence or absence of incisional hernia will also be assessed radiologically by CT routinely performed in the setting of cancer follow-up (chest-abdomen-pelvis CT with portal phase). Once the trial is completed, a second radiological review of the $\mathrm{CT}$ will be performed by a consultant radiologist. In case of discrepancy with the first radiological opinion, input from a third consultant radiologist will be requested.

\section{Secondary outcome}

The secondary outcome will be:

- The incidence of surgical site infection (SSI) at site of stoma closure, evaluated at 7 and at 31 days after stoma closure according to the Center for Disease Control definition. Specialised nurses will record pictures of the stoma site for blinded evaluation by an investigator. In case of occurrence, SSI will be treated according to institutional guidelines.

- Postoperative discomfort at stoma site, at days 7 and 31 , and 1 year after surgery, as assessed by the modified Carolinas Comfort Scale.

- Readmission linked to surgical site complications. This will be assessed over the year of postoperative follow-up.

- Reoperation linked to surgical site complications. This will be assessed over the year of postoperative follow-up.

\section{Enrolment}

Patients will be recruited during the routine preoperative consultation. Surgical teams will be briefed every 6 months about the trial, and e-mail reminders will be sent every month to ensure appropriate recruitment. Patients will be given written information related to the study and will sign the trial consent form. Trial investigators will be contacted and will provide the allocation group.

\section{Randomisation, allocation concealment and blinding}

Randomisation will be performed by JM using a 1:1 random binary sequence generated in STATA (StataCorp. V.2021. Stata Statistical Software: Release 17. College Station, Texas: StataCorp LLC) and uploaded into RedCap ${ }^{14}$ by VD, which will ensure allocation concealment. The surgery planning form will be forwarded to the investigators $(\mathrm{VD}, \mathrm{NB})$, who will indicate if a mesh should be added or not to the stoma closure procedure. Patients, radiologists and investigators performing the assessment at 1 year will be blinded for the study group. The surgical team who will perform the surgical intervention won't inform the patient about the presence of a mesh or not. Similarly, the radiological team interpreting the CT at 1 year won't be informed about the allocation group of the patients and to their participation or not in the trial. The consultant radiologist(s) verifying the CT after completion of the trial will be shown CT of the patients without knowing their allocation group and without accessing their informatised medical data. For determination of the presence or not of a clinical incisional hernia at site of stoma closure, clinical examination will be performed by a surgeon not involved in the primary procedure. In case of local complication occurring at the site of stoma closure and requiring surgery, unblinding will be performed for adequate management.

\section{Sample size calculation}

Considering the results of existing studies comparing the incidence of incisional hernia after stoma closure with and without prophylactic mesh, we will aim to reduce the incidence of incisional hernia (both clinical and radiological) from $30 \%$ to $5 \%$. Using the one-sided Pearson's $\chi^{2}$ test for proportions, with a power set at 0.8 and an alpha at 0.05 , and considering a 1:1 allocation ratio, we have to include 56 patients (28 with mesh and 28 without mesh). By estimating a $20 \%$ dropout rate, the final sample size includes 68 patients (34 with mesh, 34 without mesh). The sample size calculation was performed using STATA (StataCorp. V.2021. Stata Statistical Software: Release 17. College Station, Texas: StataCorp LLC)

\section{Variables of interest}

The investigators will record variables of interest into a case-report form generated in the RedCap software. ${ }^{14}$

\section{Variables related to stoma creation}

- Date of stoma creation

- Age

- Gender

- Body mass index (BMI)

- American Society of Anesthesiologists physical status classification system (ASA) class

- Tobacco use

- Prealbumin concentration

- Albumin concentration

- Protein concentration

- Neo-adjuvant treatment

- Surgery performed in emergency or elective setting

- Diagnostic

- Type of surgery performed

- Type of stoma

- Site of stoma

- Length of stay. 
Variables related to stoma closure

- Date of stoma closure

$\rightarrow$ Age

- BMI

- ASA class

- Tobacco use

- Prealbumin concentration

- Albumin concentration

- Proteins concentration

- Type of surgery performed

- Presence or not of a parastomal hernia

- Length of stay.

Variables related to follow-up at 7 days after the stoma closure

- Presence or not of a SSI.

- Pictures of the stoma site.

Variables related to follow-up at 31 days after the stoma closure

- Presence or not of a SSI.

- Pictures of the stoma site.

- Modified Carolinas Comfort Scale.

Variables related to follow-up at 1 year after stoma closure

- BMI

- Adjuvant treatment

- Presence or not of an incisional hernia at the site of stoma closure (clinical examination)

- Presence or not of an incisional hernia at the site of stoma closure (CT)

- Number of defect(s)

- $\operatorname{Size}(\mathrm{s})$ of the $\operatorname{defect}(\mathrm{s})$

- Content of the defect(s)

- If appropriate, variables related to the management of the incisional hernia.
PROCEDURES AT EACH VISIT

\section{Screening visit ( -2 to -3 weeks)}

The patient will be seen by a surgeon during the preoperative medical visit. Standard clinical examination and preoperative blood sampling will be performed. Informed consent will be obtained and the patient will be randomised into one of the study group. To ensure adequate recruitment and be certain to enrol all eligible participants, theatre lists will be screened by the investigators on a daily basis.

\section{Baseline visit (day 0)}

The patient will be seen by the surgeon prior to surgery, and after surgery. The patient will be blinded to the allocation group.

\section{Postoperative visit 1 (day 7)}

The patient will be seen by a specialised nurse, performing a clinical assessment of SSI at stoma site, and taking pictures. The modified Carolinas Comfort Scale questionnaire will be filled.

\section{Postoperative visit 2 (day 31)}

The patient will be seen by a specialised nurse, performing a clinical assessment of SSI at stoma site, and taking pictures. The modified Carolinas Comfort Scale questionnaire will be filled.

\section{Postoperative visit 3 (1 year)}

The patient will be seen at 1 year after the surgical intervention for clinical examination of the stoma site and interpreting the CT for signs of incisional hernia. The modified Carolinas Comfort Scale questionnaire will be filled.

The follow-up is summarised in figure 1.

\section{Statistical analysis}

Continuous variables will be expressed as means \pm SE error of the mean (SEM). Categorical variables will be

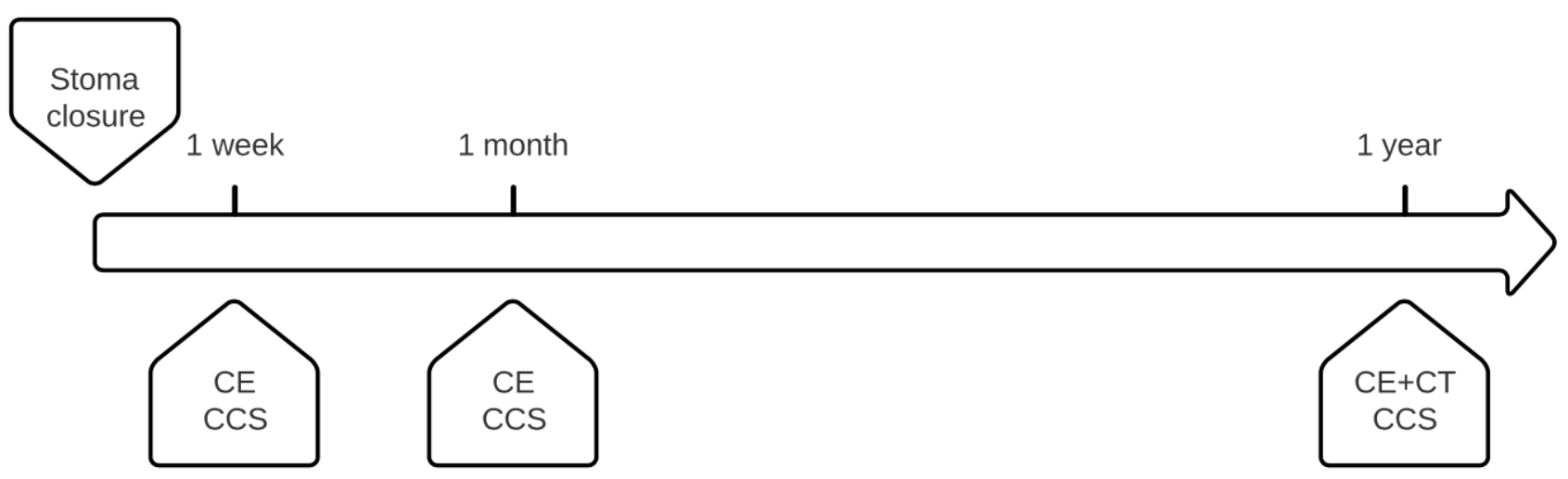

Figure 1 Follow-up after stoma closure. Clinical examination (CE) looking for surgical site infection at site of stoma closure will be performed at 1 week and at 1 month after stoma closure. The Carolina Comfort Scale (CCS) will be filled by the patients during these visits. CE and CT will be performed at year after stoma closure, looking for evidence of incisional hernia at site of stoma closure. 
expressed as absolute frequencies and percentages. Continuous variables and outcomes will be compared using the two-sided Student's t test. With more than 30 patients per group, according to the central limit theorem, the t-test remains applicable even if the data are not normally distributed. Categorical variables and outcomes will be compared using the $\chi^{2}$ test or the exact Fisher test, when appropriate. Subgroup analyses will be performed according to the WHO BMI class, the type of hernia (clinical or radiological) and the type of stoma. An interim analysis will be performed when 34 patients are included; if a difference $>15 \%$ of SSI (the short-term outcome) between the two groups is observed, the study will be terminated. A $p<0.05$ will be considered as significant. Analyses will be performed in intention-to-treat and using GraphPad Prism V.15 (GraphPad Software, USA) and STATA V.13.

\section{DISCUSSION}

Existing literature reports heterogeneous incidences of incisional hernia at stoma site after stoma closure. Several systematic reviews and meta-analyses, however, documented clinically significant pooled incidences. ${ }^{115}$ Considering that such incisional hernias usually require surgery and hospitalisation, we believe that this complication, in addition to altering patients' quality of life, also impairs healthcare systems budgets.

Preliminary studies have shown that the insertion of a prophylactic mesh, resorbable or not, during stoma closure reduces the incidence of incisional hernia at stoma site ${ }^{9-11}$. Further, the procedure seems to be safe, with low incidences of SSI reported.

The present RCT aims at determining whether the application of a prophylactic non-absorbable mesh during stoma closure allows or not for the prevention of incisional hernia at 1 year. Subgroup analysis will be performed according to the type of stoma, the type of incisional hernia and to BMI class. The primary outcome will be assessed by clinical examination and by CT, which was reported to be more sensitive in case of parastomal hernias. $^{16}$

The strengths of the present RCT will be the following: (1) good external validity due to the representation of different types of lower gastrointestinal tract stomas, (2) application of an affordable mesh in the sublay position, where the risk of intra-abdominal complications is lower and application is the easiest (as compared with intraabdominal mesh), (3) assessment of the presence or not of incisional hernia at 1 year by CT, which is more accurate than clinical examination, especially in patient suffering from obesity. The limitations of the present RCT will be the following: (1) no assessment of the long-term incidence of incisional hernia, (2) pooling of all types of stomas, which have slightly different incidences of incisional hernia after closure (however, this also constitutes a pragmatic approach giving an universal answer to clinicians), (3) monocentre design, which may affect recruitment rate and external validity.

To conclude, prevention of incisional hernia at site of stoma closure could potentially lead to an improvement in patients' quality of life and generate savings for healthcare systems. With the present RCT, we expect to demonstrate that the application of a prophylactic mesh reduces the 1-year incidence of incisional hernia at site of stoma closure.

\section{ETHICS AND DISSEMINATION}

The present RCT was registered into clinicaltrials.gov (NCT 04510558) and was accepted by the local ethic committee (Geneva, Switzerland: CCER 2021-00053). Patients' confidentiality will be ensured by coding the data. RedCap will be used for data collection and storage. ${ }^{14}$ The investigators have no conflict of interest to declare. The results of the trial will be presented at national and international congresses in the fields of colorectal surgery and general surgery, and published in a peer-reviewed journal.

\section{Trial status}

Currently recruiting.

Contributors JM, VD and NB performed the review of the literature and conceived the study. JM performed the sample size calculation. JM and VD drafted the protocol manuscript, submitted the protocol to the local ethics committee and to clinicaltrials.gov. JM, VD, ZA, JD, CT, FR and NB performed critical revision of the manuscript and accepted its final version.

Funding The authors have not declared a specific grant for this research from any funding agency in the public, commercial or not-for-profit sectors.

Competing interests None declared.

Patient and public involvement Patients and/or the public were not involved in the design, or conduct, or reporting, or dissemination plans of this research.

Patient consent for publication Not applicable.

Provenance and peer review Not commissioned; externally peer reviewed.

Open access This is an open access article distributed in accordance with the Creative Commons Attribution Non Commercial (CC BY-NC 4.0) license, which permits others to distribute, remix, adapt, build upon this work non-commercially, and license their derivative works on different terms, provided the original work is properly cited, appropriate credit is given, any changes made indicated, and the use is non-commercial. See: http://creativecommons.org/licenses/by-nc/4.0/.

ORCID iD

Jeremy Meyer http://orcid.org/0000-0003-3381-9146

\section{REFERENCES}

1 De Haes F, Bullen NL, Antoniou GA, et al. Systematic review and meta-analysis of incisional hernia post-reversal of ileostomy. Hernia 2020;24:9-21.

2 Cingi A, Solmaz A, Attaallah W, et al. Enterostomy closure site hernias: a clinical and ultrasonographic evaluation. Hernia 2008;12:401-5.

3 Cingi A, Cakir T, Sever A, et al. Enterostomy site hernias: a clinical and computerized tomographic evaluation. Dis Colon Rectum 2006;49:1559-63.

4 Mongelard K, Mynster T, Jensen KK. Stoma-site hernia after stoma reversal following rectal cancer resection. Dan Med J 2020;67.

5 van Ramshorst GH, Eker HH, Hop WCJ, et al. Impact of incisional hernia on health-related quality of life and body image: a prospective cohort study. Am J Surg 2012;204:144-50. 
6 Poulose BK, Shelton J, Phillips S, et al. Epidemiology and cost of ventral hernia repair: making the case for hernia research. Hernia 2012;16:179-83.

7 Gillion J-F, Sanders D, Miserez M, et al. The economic burden of incisional ventral hernia repair: a multicentric cost analysis. Hernia 2016;20:819-30.

8 Burger JWA, Luijendijk RW, Hop WCJ, et al. Long-term follow-up of a randomized controlled trial of suture versus mesh repair of incisional hernia. Ann Surg 2004;240:578-85.

9 Liu DSH, Banham E, Yellapu S. Prophylactic mesh reinforcement reduces stomal site incisional hernia after ileostomy closure. World $J$ Surg 2013;37:2039-45.

10 Maggiori L, Moszkowicz D, Zappa M, et al. Bioprosthetic mesh reinforcement during temporary stoma closure decreases the rate of incisional hernia: a blinded, case-matched study in 94 patients with rectal cancer. Surgery 2015;158:1651-7.

11 Reinforcement of Closure of Stoma Site (ROCSS) Collaborative and West Midlands Research Collaborative. Prophylactic biological mesh reinforcement versus standard closure of stoma site (ROCSS): a multicentre, randomised controlled trial. Lancet 2020;395:417-26.

12 Chan A-W, Tetzlaff JM, Gøtzsche PC, et al. Spirit 2013 explanation and elaboration: guidance for protocols of clinical trials. $B M J$ 2013;346:e7586

13 Schulz KF, Altman DG, Moher D, et al. Consort 2010 statement: updated guidelines for reporting parallel group randomised trials. BMJ 2010;340:c332.

14 Harris PA, Taylor R, Thielke R, et al. Research electronic data capture (REDCap)--a metadata-driven methodology and workflow process for providing translational research informatics support. J Biomed Inform 2009;42:377-81.

15 Bhangu A, Nepogodiev D, Futaba K, et al. Systematic review and meta-analysis of the incidence of incisional hernia at the site of stoma closure. World J Surg 2012;36:973-83.

16 Odensten C, Strigård K, Rutegård J, et al. Use of prophylactic mesh when creating a colostomy does not prevent parastomal hernia: a randomized controlled Trial-STOMAMESH. Ann Surg 2019;269:427-31. 